\title{
Bases pedagógicas em curso profissionalizante de Farmácia e Laboratório Clínico como apoio na construção profissional do indivíduo
}

\author{
Pedagogical bases in the professionalizing profession of pharmacy and clinical laboratory \\ as support in the professional construction of the individual
}

\section{Bases pedagógicas en el curso profesional de la Farmacia y el Laboratorio Clínico como la práctica en la construcción profesional del individuo}

Silvio de Almeida Junior ${ }^{1,2 *}$, Fernanda Carolina da Silva ${ }^{1}$, Nhaypi lasmin Taveira Moreira ${ }^{1}$, Danilo Cândido Bulgo², Leticia Natália Oliveira², Alex Alves Rodrigues ${ }^{3}$, Gabriel Henrique Vieira Silva², Cristian Ribeiro Gonçalves², Bruna Castalgini de Souza², Luiz Alexandre Pereira ${ }^{4}$, Matheus Reis Santos de Melo $^{2}$, Fernanda de Castro Nakamura ${ }^{5}$ Gessica Andrade ${ }^{2}$.

\section{RESUMO}

Objetivo: Avaliar as práticas pedagógicas no curso profissionalizante de Farmácia e Laboratório em escola de município de médio porte do interior paulista. Métodos: Realizado através de levantamento de dados fornecidos gentilmente por escola profissionalizante, a avaliação do conteúdo programático pedagógico ligado a profissionalização do curso de Farmácia e Laboratório Clínico. Resultados: Em avaliação crítica ao conteúdo pedagógico foi realizado divisão das disciplinas ministradas agrupadas em três áreas: formação básica, formação em laboratório clínico e formação em farmácia totalizando 14 módulos e as práticas relacionadas totalizando 21 atividades. As mudanças de mercado são constantes e cabe aos profissionais 0 acompanhamento destes. A profissionalização é uma das formas na qual o trabalhador busca para se manter ativo no mercado e ser capaz de competir com a concorrência. Conclusão: A partir das avaliações, frente ao conteúdo, é possível chegar à conclusão que as aulas práticas no curso profissionalizante de Farmácia e Laboratório são de grande importância na formação profissional do aluno, levando-o não só a compreender o processo como um todo, mas o desenvolvimento de um ser pensante, no qual está preparado para resoluções de problemas no cotidiano.

Palavras-chave: Cursos livres, Formação profissional, Profissionalização.

\begin{abstract}
Objective: To evaluate the pedagogical practices in the professional course of Pharmacy and Laboratory in a medium-sized school in the interior of São Paulo. Methods: This study was carried out by means of a survey of data provided by a vocational school, the evaluation of the pedagogical content related to the professionalization of the course of Pharmacy and Clinical Laboratory. Results: In a critical evaluation of the pedagogical content, the disciplines were divided into three areas: basic training, clinical laboratory training and pharmacy training, totaling 14 modules and related practices totaling 21 activities. The market changes are constant, and it is up to the professionals to follow them up. Professionalization is one of the ways in which
\end{abstract}

\footnotetext{
${ }^{1}$ Euro Anglo Cursos Profissionalizantes, Franca, São Paulo.

2Universidade de Franca, Franca, São Paulo. *E-mail: silvioalmeidajr@yahoo.com.br

${ }^{3}$ Faculdades Metropolitanas Unidas, São Paulo, São Paulo.

${ }^{4}$ Faculdade Quirinópolis, Quirinópolis, Goiás.

5Universidade Estadual “Júlio Mesquita Filho” Franca, São Paulo.
}

SUBMETIDO EM: 3/2019

ACEITO EM: 4/2019

PUBLICADO EM: 7/2019 
the worker seeks to remain active in the market and be able to compete with the competition. Conclusion: From the evaluations, in front of the content, it is possible to reach the conclusion that the practical classes in the professional course of Pharmacy and Laboratory are of great importance in the professional formation of the student, leading not only to understand the process as a whole, but the development of a thinking being, in which he is prepared for problem solving in everyday life.

Key words: Free courses, Professional training, Professionalization.

\section{RESUMEN}

Objetivo: Evaluar las prácticas pedagógicas en el curso profesionalizante de Farmacia y Laboratorio en escuela de municipio de mediano porte del interior paulista. Métodos: Realizado a través del levantamiento de datos proporcionados gentilmente por escuela profesionalizante, la evaluación del contenido programático pedagógico ligado a la profesionalización del curso de Farmacia y Laboratorio Clínico. Resultados: En evaluación crítica al contenido pedagógico se realizó la división de las disciplinas ministradas agrupadas en tres áreas: formación básica, formación en laboratorio clínico y formación en farmacia totalizando 14 módulos y las prácticas relacionadas totalizando 21 actividades. Los cambios de mercado son constantes y corresponde a los profesionales el acompañamiento de éstos. La profesionalización es una de las formas en que el trabajador busca mantenerse activo en el mercado y ser capaz de competir con la competencia. Conclusión: A partir de las evaluaciones, frente al contenido, es posible llegar a la conclusión que las clases prácticas en el curso profesionalizante de Farmacia y Laboratorio son de gran importancia en la formación profesional del alumno, llevándolo no sólo a comprender el proceso como un todo, pero el desarrollo de un ser pensante, en el cual está preparado para resoluciones de problemas en el cotidiano.

Palabras clave: Cursos libres, Formación profesional, Profesionalización.

\section{INTRODUÇÃO}

A crise econômica vivenciada no país, leva os jovens a buscarem uma profissionalização rápida e ingresso no mercado de trabalho de forma efetiva. Para a população mais adulta, a profissionalização é um sonho buscado na intenção de trocar de área de atuação dentro do mercado de trabalho cada vez mais competitivo. As mudanças de mercado são constantes e cabe aos profissionais o acompanhamento destes. A profissionalização é uma das formas na qual o trabalhador busca para se manter ativo no mercado e ser capaz de competir com a concorrência. Empresas cada vez mais seletivas e profissionais cada vez mais preparados é o cenário atual vivido (GONDIM SE, 2002)

Assim, a formação profissional no Brasil, vem se expandindo ao longo das décadas, porém em seu início foi entendida como uma modalidade pedagógica destinada somente às classes populares, aos caboclos, pobres, escravos e índios, que deveriam ocupar-se do "saber-fazer" e de quem dependeria o conforto da vida diária. Em meados dos anos 70, a Lei 5692/71 atribuiu a compulsoriedade do ensino profissionalizante (ou técnico) em nível de segundo grau. A partir de então, todos os estudantes terminariam o ensino médio com um certificado de capacitação profissional (ROBERTO SMG et al., 2010).

Segundo o último Censo Escolar do ano de 2016 do Instituto Nacional de Estudos e Pesquisas Educacionais Anísio Teixeira (INEP), o número de estudantes inseridos nos cursos técnicos aumentou significativamente em nove anos, entre os anos de 2008 e 2016, o número passou de 1.143 milhão para 1.859 milhão no período avaliado (INEP, 2016).

Em sua expansão, os cursos profissionalizantes eram permeados pela visão "bancária" da educação, o "saber" é uma concessão dos que se julgam sábios (professores) aos que julgam nada saber (estudantes). 0 educador, era o detentor do poder, dominando o saber, enquanto os estudantes eram sempre os que não sabem. O autoritarismo e a rigidez destas posições negam a educação e o conhecimento como processos de busca (FREIRE P, 1997). 
Conforme o aumento de demanda por profissionais cada vez mais capacitados e preparados para as diversas situações vividas, cresce a procura por cursos rápidos. Sendo assim, concomitantemente, cresce também o número de escolas profissionalizantes. A profissionalização é caracterizada pela formação rápida e prática de jovens e adultos, porém o que é visto no cotidiano são escolas presas em metodologias convencionais/tradicionais em sala de aula. Cada vez mais a realização de práticas objetivada pelo uso de tecnologia se mostra eficiente desde que empregada de forma correta. Práticas pedagógicas embasadas em métodos tradicionais tendem a ser classificadas como antigas e caírem em desuso (BARDAGI M et al., 2006; FISCHER RMB, 2007).

Baseado no que é encontrado na literatura e acompanhando a constante evolução do mercado de trabalho, este artigo tem como objetivo avaliar as práticas pedagógicas implantadas no curso profissionalizante de farmácia e laboratório de uma escola localizada em município de médio porte do interior paulista. Como justificativa para realização deste, é possível citar a falta de trabalhos realizados dentro da área de ensino profissionalizante no qual é cada vez mais crescente.

\section{MÉTODOS}

\section{Caracterização do ambiente}

O trabalho foi desenvolvido em unidade de escola profissionalizante em uma cidade de médio porte do interior de São Paulo. A escola está instalada na cidade há sete anos, e gera conhecimentos profissionalizantes nas áreas de tecnologia (informática básica, avançada, hardware), idioma (inglês), gestão (empresarial e jurídica) e saúde (farmácia e laboratório, veterinária e cuidador de idosos). Os cursos são de formato livre, com duração entre seis meses a dois anos. O projeto foi desenvolvido entre os meses de junho de 2017 a setembro de 2018, em que contou com inclusão de 15 turmas do Curso Profissionalizante de Farmácia e Laboratório.

\section{Delineamento experimental}

Para elaboração do estudo, foi realizado avaliação pedagógica do conteúdo programático do curso de Auxiliar de Farmácia e Laboratório. O curso tem a duração de 14 meses, e são subdivido em módulos mensais. Após a análise pedagógica, foi proposto a inclusão de práticas de cunho profissionalizante para agregar valores a formação dos alunos. A intenção de atividades práticas envolve o aluno de forma constante e melhora o desenvolvimento cognitivo destes, levando a uma prática de ensino-aprendizagem mais efetivo.

\section{RESULTADOS E DISCUSSÃO}

A Lei de Diretrizes e Bases da Educação Nacional - LDBEN - surge no cenário da educação atual definindo, entre seus escopos, o estímulo ao conhecimento dos problemas decorrentes do mundo contemporâneo e a prestação de serviço especializado à população, estabelecendo com ela uma relação de reciprocidade.

Tais benefícios foram reafirmadas pelas Diretrizes Curriculares, para a maioria dos cursos da área de saúde, acolhendo a importância do atendimento às demandas sociais com destaque para o Sistema Único de Saúde - SUS. Nesta perspectiva, as instituições formadoras de profissionais vêm se transformando suas práticas pedagógicas, em uma tentativa de se aproximarem da realidade social e de motivarem seus professores e estudantes a transformarem a base de conhecimento (BRASIL, 1996)

Em avaliação crítica ao conteúdo pedagógico foi realizado divisão das disciplinas ministradas (tabela 1) agrupadas em três áreas: formação básica, formação em laboratório clínico e formação em farmácia, e práticas pedagógicas para formação profissional (tabela 2) para construção do saber do indivíduo.

Dentro da formação ética (tabela 2, item a e $b$ ) houve em sala de aula, reporte de discussões frente a casos reais e demonstrados no cotidiano. A partir das práticas, é possível intensificar a formação do indivíduo na visão humanística afim de garantir a integridade no atendimento a pessoas assistidas pelo sistema de saúde, seja eles em farmácias, clínicas, laboratórios entre outros (CECCIM RB, FEUERWERKER LCM, 2004). 
Tabela 1 - Áreas de formação do curso de Auxiliar de Farmácia e Laboratório. Disciplinas ministradas durante o curso de Auxiliar de Farmácia e Laboratório de Escola Profissionalizante de cidade do interior paulista.

Formação Básica comum

Laboratório Clínico

Farmácia
Ética e bioética

Biossegurança

Gestão em saúde

Ciências Morfológicas

Microbiologia

Primeiros Socorros

Patologia

Hematologia

Análises Clínicas

Farmácia e Drogarias

Farmacologia

Cosmetologia e Estética

Fitoterapia

Toxicologia

Fonte: Dados do autor, 2018.

Tabela 2 - Práticas pedagógicas ministradas relacionando com disciplina cursada para construção da profissionalização do indivíduo, ministradas durante o curso de Auxiliar de Farmácia e Laboratório de Escola Profissionalizante de cidade do interior paulista.

\begin{tabular}{l|l}
\hline \multicolumn{1}{c}{ Prática } & \multicolumn{1}{c}{ Formação } \\
\hline a) Discussão de questões éticas & Ética e bioética \\
\hline b) Exibição de filme & Biossegurança \\
\hline c) Avaliação de espaço quanto a questões de biossegurança & Ciências Morfológicas \\
\hline d) Avaliação de pH de substâncias aquosas & Microbiologia \\
\hline e) Criação de células 3D & Primeiros Socorros \\
\hline f) Visita Técnica a Laboratório de Anatomia & Patologia / Hematologia \\
\hline g) Lavagem de mãos & Hematologia \\
\hline h) Simulação de socorro & \\
\hline i) Casos clínicos * & Análises Clínicas \\
\hline j) Esfregaço sanguínea & \\
\hline k) Tipagem sanguínea & \\
\hline l) Análise Físico-química de urina & Farmácia e Drogaria \\
\hline m) Coleta de sangue & Cosmetologia e Estética \\
\hline n) Coleta de materiais biológicos & Fitoterapia \\
\hline o) Teste de imunocromatografia & Toxicologia \\
\hline p) Aferição de pressão arterial & \\
\hline q) Aferição de glicemia capilar & \\
\hline r) Limpeza de pele & \\
\hline s) Esfoliação & t) Extração de compostos aquoso \\
\hline u) Teste de germinação com medicamentos &
\end{tabular}

Fonte: Dados do autor, 2018.

Corroborando com a evolução das práticas pedagógicas, entende-se que novas metodologias de ensino se tornam um desafio, pois exigem do professor a escolha de estratégias pedagógicas que possibilitem a participação ativa do estudante no processo de aprendizagem, assim é relevante que os professores conheçam e busquem por atividades pedagógicas, ou estejam em criação de novas situações de ensino, pois 
não há um método melhor do que outro para abordar todos os conteúdos propostos, porém é importante saber combinar os mais variados métodos para despertar o interesse do estudante, aumentando as possibilidades de aprendizagem a fim de atingir os objetivos propostos (DIAZ BJ, 2007).

Dentro do módulo de biossegurança, são realizadas atividades assistidas para construção do conhecimento prático. Para assegurar isso, são realizadas visitas técnicas a estabelecimentos de saúde (ou não) afim de avaliarem a estrutura física quanto as normas de biossegurança (tabela 2, item $c$ ), levando em consideração o tipo de trabalho e os tipos de riscos no qual o trabalhador é exposto. Como propósito, as avaliações de riscos servem para identificar o conjunto de procedimentos afim de avaliar produção, produtos e resíduos (HÖKERBERG YHM, et al., 2006).

As práticas são destinadas a responder questionamentos cotidianos, gerados pelos próprios alunos. Frente a isso, uma prática instalada é a realização da aferição do potencial hidrogeniônico $(\mathrm{pH})$ (tabela 2, item d) levando ao conhecimento do que é ácido, neutro ou alcalino. A aferição do pH é importante em indústrias farmacêuticas, hospitais e laboratórios sendo aplicados na rotina de trabalho. A atividade leva ao conhecimento e prática, visto que a leitura muitas vezes é realizada de forma manual, sendo a acuidade visual a forma de identificação.

Assim como é visto em todos os cursos dentro da área da saúde, a base curricular em ciências morfológicas (embriologia, citologia, histologia e anatomia) é de extrema importância para formação do profissional. Toda a base se inicia pela construção e funcionamento de um organismo vivo. O conhecimento para formação do corpo humano (tabela 2, itens e e $f$ ), conta com a construção de informações em 3D faz com que o aluno tenha ideia das dimensões reais de uma célula ou tecido e vai além, quando os alunos realizam visita técnica a laboratório de ciências morfológicas (anatomia) de uma Universidade parceira da escola para o estudo em cadáveres preservados. A informação visual é de suma importância para fixação do conteúdo (MONTANARI T e BORGES E, 2013).

Durante o curso, são tratadas informações pertinentes a contaminação e infecções hospitalares. Por isso, a prática de lavagem de mãos (tabela 2, item $\mathrm{g}$ ) compreende a forma correta e organizada de higienização, para evitar transmissão de contaminantes. Durante a prática, o docente, leva o aluno a compreender a importância da lavagem correta das mãos e principalmente, o risco que isso pode acarretar na exposição a pacientes, incluindo aumento de tempo de internação, aumento do custo, demanda de medicação e cuidados e até o óbito (NOGUERAS M, et al., 2001).

Quanto ao assunto primeiros socorros, é visto a pouca difusão quando se orienta com informações de acidentes ocorridos em locais públicos ou até mesmo em domicílios. Frente a isso, o propósito das práticas do módulo é orientar sobre as principais formas de primeiros socorros (tabela 2, item $h$ ). As aulas são expositivas, de forma prática, que leva ao aluno a vivenciar possíveis intercorrências durante o dia-a-dia. Compreende-se que, ao tratar metodologias ativas e ensino prático apoiado ao teórico eleva o interesse e dinamismo das práticas, levando a melhor compreensão do assunto (VERONESE AM et al., 2010).

Um dos diferenciais implantados na construção profissional dos alunos de Farmácia e Laboratório são os estudos de casos clínicos (tabela 2, item 1). A construção do conhecimento através de metodologia ativa adaptada trouxe grandes benefícios aos alunos. Em cursos superiores são utilizados o PBL (Problem Based Learning) que no português quer dizer: Aprendizagem baseada em problemas. A metodologia já é bastante empregada em cursos de saúde (medicina, odontologia, farmácia, enfermagem, nutrição, entre outros). Os resultados obtidos são promissores, visto que o método de ensino ativo prepara melhor o aluno e o condiciona a sempre estudar para que responda a questionamentos realizados no cotidiano. Dentro da literatura, não há indícios do método real ou adaptado utilizado em cursos profissionalizante, fazendo com que o presente trabalho seja pioneiro nessa área (BERBEL NAN, 1998; CYRINO EG e TORALLES ML, 2004; REGO S, 1998; REIS P, 2007).

Compreendendo que o curso tem finalidade de lançar o aluno ao mercado de trabalho, as práticas são programadas na construção do profissional preparado, incluindo as atividades destinadas a laboratórios clínicos (tabela 2, itens $j, k, l, m, n$ e o) e as avaliações de parâmetros fisiológicos e bioquímicos (tabela 2, $p$ 
e q) levam ao treinamento e destreza de manipulação de materiais com a finalidade de garantir a qualidade e segurança dos pacientes assistidos por esses futuros profissionais. A garantia disso, é assegurada pelos docentes em sala de aula e confirmado através de comprovação teórica em atividades avaliativas.

Em práticas paralelas, na intenção de introduzir conhecimento técnico-científico aos alunos, são realizados dentro da área de estética e cosmetologia (esfoliação e limpeza de pele, descritos na tabela 2, itens $r$ e $s$ ). São práticas simples, sem a utilização de procedimentos invasivos, que podem ser reproduzidos em casa. Essas atividades são avaliadas como benéficas e não trazem riscos à saúde. Ainda, o pensar científico é levado através das práticas de extração de compostos por métodos de maceração (tabela 2, item $t$ ) obtendo um extrato aquoso. A extração de extratos é comum na prática farmacêutica. A fim de gerar conhecimentos, esses extratos, são testados quanto ao seu potencial toxico em germinação (tabela 2, item $u$ ).

\section{CONCLUSÃO}

A partir das avaliações frente ao conteúdo, é possível chegar à conclusão que as aulas práticas no curso profissionalizante de Farmácia e Laboratório de escola de município de médio porte do interior paulista são de grande importância na formação profissional do aluno, levando-o não só compreender o processo como um todo, mas o desenvolvimento de um ser pensante, no qual está preparado para resoluções de problemas no cotidiano. Tal resposta corrobora com dados achados em bases de dados, no qual compreende que a diversidade de conceitos adquiridos amplia o conhecimento e é dado como melhor forma de aprendizado.

\section{AGRADECIMENTOS}

O presente trabalho foi realizado com apoio da Coordenação de Aperfeiçoamento de Pessoal de Nível Superior - Brasil (CAPES) - Código de Financiamento 001.

\section{REFERÊNCIAS}

1. BARDAGI M, et al. Escolha Profissional e Inserção no Mercado de Trabalho: Percepções de Estudantes Formandos. Psicol. Esc. E Educ., 2006; 10, 69-92.

2. BERBEL NAN. A problematização e a aprendizagem baseada em problemas: diferentes termos ou diferentes caminhos? Interface - Comun. Saúde Educ., 1998; 139-154.

3. BRASIL. Lei oㅜ 9.394 de 20 de dezembro de 1996. Estabelece as diretrizes da Educação Nacional. Diário Oficial da União 23 dez. 1996;

4. CECCIM RB, FEUERWERKER LCM. O quadrilátero da formação para a área da saúde: ensino, gestão, atenção e controle social. Physis Rev. Saúde Coletiva, 2004; 14, 41-65.

5. CYRINO EG, TORALLES PML. Trabalhando com estratégias de ensino-aprendizado por descoberta na área da saúde: a problematização e a aprendizagem baseada em problemas. Cad. Saúde Pública, 2004; 20, 780-788.

6. DIAZ BJ, PEREIRA AM. Estratégias de ensino-aprendizagem. 28a ed. Petrópolis: Vozes; 2007; 312p.

7. FISCHER RMB. Mídia, máquinas de imagens e práticas pedagógicas. Rev. Bras. Educ., 2007; 12, 290-299.

8. FREIRE P. Educação "bancária" e educação libertadora. Introdução à psicologia escolar, v. 3, p. 61-78, 1997

9. GONDIM SMG. Perfil profissional e mercado de trabalho: relação com formação acadêmica pela perspectiva de estudantes universitários. Estud. Psicol. Natal, 2002; 7, 299-309.

10. HÖKERBERG YHM, et al.; O processo de construção de mapas de risco em um hospital público. Ciênc. Saúde Coletiva, 2006; 11, 503-513.

11. INEP - Educação profissional técnica de nível médio no censo escolar. Disponível em <http://portal.inep.gov.br/informacao-dapublicacao/-/asset_publisher/6JYlsGMAMkW1/document/id/489599> Acesso em 10 jun. 2019

12. MONTANARI T, BORGES E. Museu Virtual do Corpo Humano: Ambiente Virtual de Aprendizagem para o Ensino de Ciências Morfológicas. RENOTE; 2013; 10, 1-11.

13. NOGUERAS M. et al. Importance of hand germ contamination in health-care workers as possible carriers of nosocomial infections. Rev. Inst. Med. Trop. São Paulo, 2001; 43, 149-152.

14. REGO S. Currículo paralelo em Medicina, experiência clínica e PBL: uma luz no fim do túnel? Interface - Comun. Saúde Educ., 1998; 2, 35-48.

15. REIS P. O ensino da ética nas aulas de ciências através do estudo de casos. Interacções, 2007; $36-45$.

16. ROBERTO SE, et al. O efeito do ensino profissionalizante sobre a probabilidade de inserção no mercado de trabalho e sobre a renda no período pré-planfor. Ver. EconomiA, 2010; 11, 155-174.

17. VERONESE AM, et al. Oficinas de primeiros socorros: relato de experiência. Rev. Gaúcha Enferm 2010; 31, 179-182. 